\title{
A New Forum for DOHaD
}

Welcome to the first issue of Journal of Developmental Origins of Health and Disease (J DOHaD). The field of developmental origins, or fetal programming as it is sometimes known, has generated tremendous interest during the past decade, culminating in the demand for this journal. Whereas medicine has classically focused upon disease states, often associated with infection or aging, we now recognize that processes during development, ranging from preconception through adolescence, may predispose or prevent susceptibility to disease pathologies. Evidence for the critical influence of developmental origins spans human epidemiologic studies, animal physiology, cellular and molecular experiments, and epigenetic mechanisms. The implications of $\mathrm{DOHaD}$ have already influenced public policy and health economics. J DOHaD offers the opportunity to provide a forum representing both the breadth of studies and depth of science elucidating this field. As such, the Journal will provide a broad based representation of studies pertinent to $\mathrm{DOHaD}$. Each Issue will include several Reviews, as well as Original Articles incorporating human, animal, and cellular/ molecular models. We will publish Commentaries on select papers, as evidenced in the current Issue, and further encourage our readers to respond with Letters to the Editor.

We further anticipate the development of themed Issues and Supplements which may represent proceedings of meetings relevant to the $\mathrm{DOHaD}$ theme. Individuals interested in themed Issues or organizations interested in supplements are invited to contact me.

$J \mathrm{DOHaD}$ represents the flagship publication of the $\mathrm{DOHaD}$ Society. Accordingly, each Issue will contain news items and schedules of upcoming conferences of interest to our readership. The Journal also provides an opportunity for communication, as evidenced in the current Issue with the statement of Dr Mark Hanson, DOHaD Society President.

The Journal owes a great debt of gratitude to the contribution of our Associate Editors, Editorial Board, and the commitment already exhibited by our reviewers and submitting authors. Our Editorial Board represents an international multi-disciplinary Board of extraordinary investigators, which underscores the focus of our Journal. Of note, Associate Editors and Editorial Board members include members and non-members of DOHaD Society. As Associate Editors and Editorial Board members will rotate their terms of service, we anticipate opportunities for new individuals to participate in the continued development and progress of the Journal. Those individuals interested in a present or future role with the Journal are invited to contact me.

The Journal is dedicated to rapid review and correspondence related to submissions, and we aim to publish papers online within 4-6 weeks of acceptance. We further have an opportunity for Rapid Communications, and encourage submission of all articles meeting the criteria laid out in the Instructions for Contributors accessed via our homepage (journals.cambridge.org/doh).

Importantly, we have been pleased to partner with Cambridge University Press, and thus benefit from Cambridge Journals Online, an industry-leading platform. Cambridge Journals Online is regularly updated with innovative new features and offers authors high visibility for their work. We encourage you to visit our homepage to find out more about the Journal, read articles published online ahead of print under the FirstView section, and sign up to receive free alerts to let you know when the latest papers have been published.

With this exciting start, we recognize that the Journal can only succeed with the continued support and enthusiasm of our authors and readership. I personally look forward to hearing from you regarding your comments, feedback, or suggestions for Journal advances at the email addresses below.

Michael G. Ross, M.D., M.P.H. Editor-In-Chief

DOHaDEditor@cambridge.org DOHaDAssistant@cambridge.org 